\title{
Brief Education to Promote Maternal Influenza Vaccine Uptake: A Randomized Controlled Trial
}

WONG Wing Yu, Valerie ${ }^{\mathrm{a}}$

FONG Yee Tak, Daniel ${ }^{\mathrm{a}}$

LOK Yuet Wan, Kris ${ }^{\mathrm{a}}$

WONG Yuen Ha, Janet ${ }^{\mathrm{a}}$

SING Chu ${ }^{\mathrm{b}}$

CHOI Yin-yin, Alice ${ }^{c}$

YUEN Yuet Sheung, Carol $^{\mathrm{a}}$

Marie TARRANT, as

${ }^{a}$ School of Nursing, The University of Hong Kong, 21 Sassoon Road, Pokfulam, Hong Kong ${ }^{\mathrm{b}}$ Dept. of Obstetrics and Gynaecology, Kwong Wah Hospital, 25 Waterloo Road, Kowloon, Hong Kong

${ }^{c}$ Dept. of Obstetrics and Gynaecology, Queen Mary Hospital, 102 Pokfulam Road, Pokfulam, Hong Kong

\section{${ }^{\S}$ Corresponding Author Contact Information:}

Marie Tarrant

Associate Professor, School of Nursing

4/F, William M. W. Mong Block

Li Ka Shing Faculty of Medicine

21 Sassoon Road, Hong Kong

Tel: +85239176643

Fax: +85228726079

Email: tarrantm@hku.hk

Co-author Email Addresses:

VW: valywong@gmail.com

DF: dytfong@hku.hk

KL: krislok@hku.hk

JW: janetyh@hku.hk

SC: singc@ha.org.hk

AC: choiyy@ha.org.hk

CY: carol_iou@hotmail.com

Word Count: 3530 words

Clinical Trial Registration: www.clinicaltrials.gov (NCT01772901). 


\section{1. Introduction}

2 Pregnant women have higher rates of influenza-related hospitalizations [1], complications [2,

3 3], and mortality $[2,4]$ during pandemic and non-pandemic years. Influenza vaccination is

4 beneficial and safe for pregnant women throughout pregnancy [5-8] and provides protection

5 for the newborn in the first 6 months of life [9]. Although the World Health Organization

6 (WHO) has identified pregnant women as having highest priority for seasonal influenza

7 vaccination [10], maternal influenza vaccination rates are often lower than in other high-risk

8 groups and the general population [11-13]. A recent review of influenza vaccination rates in

9 pregnant women across 11 countries found vaccination rates ranged from $1.7 \%-88 \%$, but

10 were most often less than $50 \%$ [14].

11 Pregnant women who have more knowledge about the potential complications of

12 influenza and the safety of the influenza vaccine are more likely to be vaccinated [15-17]. To

13 date, the majority of interventions aimed at improving maternal influenza vaccination rates

14 have targeted healthcare providers, primarily obstetricians, and encouraged them to discuss

15 influenza vaccination with pregnant women [18-22]. Among pregnant woman-focused

16 interventions, one trial showed that an education pamphlet, with or without a verbalized

17 benefits statement, increased vaccination rates [23]. In other studies, one found that 5 weekly

18 text messages to pregnant women about the importance of maternal influenza vaccination

19 significantly increased vaccine uptake [24] while another found that 12 weekly text messages

20 had no effect on maternal vaccination rates [25]. Chamberlain et al. [26] found that a multi-

21 component vaccination promotion intervention consisting of provider to patient education,

22 educational brochures, and an electronic patient-centred tutorial did not improve vaccine

23 uptake. Frew et al. [27] evaluated the effect of two types of vaccination messages (i.e.,

24 information about the benefits of vaccination vs. information about the risks of not

25 vaccinating) and found that neither of the two message types significantly improved

26 vaccination uptake. 
The low rate of vaccine uptake in this target group and the conflicting evidence from evaluated interventions indicate a need to further develop interventions to improve maternal influenza vaccine rates. Although the Hong Kong government has endorsed the WHO recommendation for prioritizing pregnant women in seasonal and pandemic influenza

vaccination programs, there is no free or subsidized vaccination program for this target group and publicly-funded antenatal clinics do not provide influenza vaccination as part of routine care to pregnant women. Pregnant women must get vaccinated in private clinics, primarily general practice clinics dispersed throughout the city. In public antenatal clinics, pregnant women do not have a dedicated provider and at each visit are assessed by a midwife or physician, depending on their stage of pregnancy and any complicating conditions. Thus, provider-focused interventions would likely be ineffective in such settings and interventions targeting pregnant women may be more appropriate to improve influenza vaccination coverage. The objective of the present study was to assess the effect of a brief education intervention targeting pregnant women on the uptake of influenza vaccination.

\section{Materials and Methods}

\subsection{Design, setting, and participants}

We designed a randomized controlled trial to evaluate the efficacy of a brief, one-to-one education session on the influenza vaccination rate during pregnancy and the proportion of participants seeking out influenza vaccination. A more detailed study protocol is reported elsewhere [28]. During two consecutive influenza seasons (2013-14 and 2014-15), pregnant women attending the antenatal clinics at four geographically-dispersed public hospitals in Hong Kong were screened for eligibility and recruited into the study by a research nurse. These hospitals were selected based on geographical representativeness and the large populations of eligible pregnant women from a wide range of socioeconomic backgrounds they served. Hong Kong has eight public and ten private hospitals that offer obstetric services. 
Public health care, including high-quality antenatal, postnatal and well-child health care, is available free of charge to all Hong Kong residents. Private health care is available on a fee for service basis. In 2011, two-thirds of all Hong Kong women gave birth in public hospitals [29]. Although women giving birth in private hospitals are usually of higher socioeconomic status, many high-income families chose to access public maternity services because it is free, high quality and comprehensive.

Inclusion criteria were pregnant women: (a) with a singleton pregnancy; (b) at least 18 years of age; (c) in at least the second trimester of pregnancy; (d) who spoke Cantonese; (e) were Hong Kong residents; (f) without serious medical conditions (i.e., cancers, rheumatoid arthritis, major psychiatric illnesses) or obstetrical complications (i.e., full placenta previa or diagnosed birth defects); (g) who had not yet received the influenza vaccination during this pregnancy; and (h) who would be staying in Hong Kong for at least 2 weeks after birth. Nonresidents who are not entitled to health benefits in Hong Kong were excluded. Although influenza vaccine is safe in any trimester of pregnancy, we recruited pregnant women after the first trimester to avoid any perceived association between vaccination and early pregnancy complications.

\subsection{Randomization and concealment}

Participating pregnant women were randomized into either a standard care group or an intervention group at a 1:1 ratio, using block randomization with random block sizes of 2-8. An independent researcher who did not participate in the study generated an allocation sequence using Stata 13.1 statistical software (StataCorp 2013, Stata Statistical Software: Release 13, College Station, TX; StataCorp LP). Treatment assignments were placed in sequentially numbered, sealed, opaque envelopes. The research nurse selected the next envelope in the sequence to determine treatment allocation, after the eligible pregnant women 
were given information about the study and had signed a written consent form. Blinding of the research nurse and participants was not possible given the nature of the intervention.

\subsection{Intervention}

Standard antenatal care consists of routine checking of maternal and fetal health by either obstetricians or midwives, along with health education to promote a healthy pregnancy.

Childbirth preparation classes were available to all women attending the clinics for no additional cost. Recommendations and education about influenza vaccination in pregnancy are not normally included in routine antenatal care. However, participants allocated to the standard care group were provided with an education pamphlet on influenza vaccination in pregnancy, developed by the Hong Kong Centre for Health Protection (CHP) [30] and freely available in the antenatal clinics during the study.

The intervention group received standard care plus brief one-to-one education lasting 10 minutes that focused on four key recommendations identified from the literature: (i) informing the participants about vaccination recommendations; (ii) encouraging them to discuss vaccination with their antenatal care provider or general practitioner (GP); (iii) increasing accessibility of the vaccine by referral to clinics where vaccination could be obtained; and (iv) providing influenza-related information from the official government website and the website uniform resource locator [14]. Specifically, participants in the intervention group were informed about: (i) the WHO [10] and Hong Kong CHP recommendations [31] regarding influenza vaccine during pregnancy; (ii) potential complications associated with influenza infection during pregnancy and for young infants; (iii) the safety of influenza vaccination for pregnant women; (iv) potential benefits of influenza vaccination for pregnant women and infants; and (v) where and how to get the influenza vaccination in Hong Kong. Almost all participants had a personal GP who provided 
influenza vaccine and for the few that did not, we provided information on nearby clinics that

could provide vaccination.

Immediately after randomization, the intervention was delivered in a private room in

the antenatal clinics so that participants in the standard care group were unable to overhear the

other participants received. A digital flip chart was used to present the education content and

participants were encouraged to express concerns and ask questions. To ensure consistency of intervention delivery, one research nurse carried out the education intervention across the four

111 sites.

\subsection{Data collection}

114 All participants completed a standard baseline questionnaire collecting: (i) key background data (i.e., age, marital status, education level, family income, and employment status); (ii) maternal health status (i.e., pre-existing health conditions, pregnancy-related health problems,

117 gravidity and parity, and expected date of confinement); and (iii) influenza and influenza 118 vaccine knowledge. Participants were subsequently followed up by telephone at 2-3 weeks

119 after their expected delivery date by a study research assistant who had not been involved in 120 participant recruitment and was blinded to participants' treatment allocation. During the 121 follow-up telephone interviews, participants reported their influenza vaccination status during 122 the pregnancy, reasons for receiving or not receiving influenza vaccination, discussion of

123 influenza vaccination with antenatal care providers or GPs, attempts to receive the

124 vaccination (i.e., participant went to their GP and requested the vaccine but were unable to receive it), and anti-vaccination advice from any healthcare professional. 
128 The primary study outcome was the self-reported influenza vaccination rate during pregnancy.

129 The secondary outcomes were the proportion of participants who initiated discussion about

130 influenza vaccination with a healthcare professional and the proportion of participants who

131 attempted to get vaccinated.

132

133

\subsection{Sample size calculation}

134 Previous Hong Kong studies showed that seasonal influenza vaccination uptake among 135 pregnant women ranged from $1.7 \%-5 \%[15,32,33]$. Other studies also showed that in 136 pregnant woman-focused interventions, the risk difference of influenza vaccination uptake 137 among pregnant women before and after implementing the intervention ranged from $2 \%$ to $13839 \%[23-25,27]$. Therefore, an estimate of the "normal" influenza vaccination uptake rate 139 among pregnant women in Hong Kong would be $5.0 \%$, and an increase to $20 \%$ would be 140 conservative but clinically meaningful. With a power of 0.80 and significance level of 0.05 141 and using a chi-square test in the G-power statistical analysis program [34], we calculated that 14276 participants would be required for each group (152 participants in total). After accounting 143 for a loss to follow-up and dropout rate of around 20\%, approximately 92 participants per 144 group were required, giving a total of 184 participants.

\subsection{Data analysis}

147 Baseline sociodemographic characteristics of the two groups were compared using a $\chi^{2}$ test or 148 a Fisher's Exact Test for categorical variables and Student's t-test for continuous variables.

149 The proportion of participants in the two study groups who received influenza vaccination 150 during pregnancy was compared using $\chi^{2}$ tests. We further computed the odds ratios of 151 vaccination using logistic regression, while adjusting for one baseline variable that was 152 significantly different between the two groups. The intention-to-treat principle was used, with 
153 missing values taken as no vaccination while the per-protocol analysis, with missing values

154 removed, was reported as a comparison. We used $\chi^{2}$ tests to compare the proportion of

155 participants in the two groups who discussed influenza vaccination with a healthcare

156 professional and the proportion of participants who attempted to receive influenza vaccination.

157 Each estimate was accompanied by a 95\% confidence interval (CI); a 5\% level of significance

158 was considered statistically significant in all statistical tests. Data analyses were performed

159 using Stata statistical software (StataCorp 2015, Stata Statistical Software: Release 14.1,

160 College Station, TX; StataCorp LP) [35].

161

162

2.8 Ethical approval

163 Ethical approval for the study was obtained from: (1) the Institutional Review Board of the

164 University of Hong Kong/ Hospital Authority Hong Kong West Cluster; (2) the Kowloon

165 West Cluster Research Ethics Committee (KWC-REC); and (3) the Ethics Committee of

166 Hong Kong East Cluster (EC-HKEC). Informed written consent was obtained from all study

167 participants before any personal data were collected and the intervention delivered. The

168 research nurse informed each eligible pregnant woman about the purpose and nature of the

169 study, the potential benefits and risks of participation, and their right to refuse to participate or

170 withdraw at any time during the study without affecting the antenatal care they received.

172 3. Results

173 Data were collected from October 7, 2013, to February 4, 2014 (Year 1), and from October 20, 1742014 , to December 23, 2014 (Year 2) (Figure 1). Data collection was interrupted in the first 175 year when several cases of H7N9 avian influenza were admitted to Hong Kong public

176 hospitals. Because of the raised influenza threat level, non-essential clinical duties were 177 suspended in all public hospitals from December 7, 2013, through January 19, 2014. 
Therefore, to achieve the required sample size, recruitment was resumed in the next influenza season. In total, 489 pregnant women were assessed for eligibility across all sites (Figure 1). Of these, $6 \%(n=29)$ did not meet the eligibility criteria, and $29 \%(n=140)$ declined to participate. Of the 321 who consented to participate, 160 were randomized to the standard care group and 161 to the intervention group; 305 (95\%) participants completed follow-up. Nine participants were lost to follow-up, and seven were contacted but refused to complete follow-up. The treatment fidelity rate was $100 \%$, because the intervention was delivered immediately after randomization.

An overview of participants' characteristics is presented in Table 1. The two groups were similar, except for a significantly higher proportion of participants with a pre-existing chronic illness in the intervention group $(\mathrm{p}=0.006)$. The reported pre-existing chronic illnesses were Hepatitis B carrier status $(n=14)$, respiratory disease $(n=6)$, thyroid disease $(n=6)$, and others $(n=13)$. The influenza vaccination rate for all participants was $15.6 \%(n=50)$ with a higher proportion of vaccinated participants in the intervention group $(21.1 \%, \mathrm{n}=34)$ than the standard care group $(10 \%, \mathrm{n}=16)$ (risk difference $[\mathrm{RD}] 11.1 ; 95 \%$ CI 3.3-19.0; $\mathrm{p}=0.006)$ (see Table 2). The number needed to treat was 9 (95\% CI 5.3-30.4). After excluding those lost to follow-up, $22.5 \%(n=34)$ of participants in the intervention group received vaccination compared with $10.4 \%(n=16)$ in the standard care group (RD 12.1\%; 95\% CI 3.9-20.3; $\mathrm{p}=0.004)$. The logistic regression analysis showed that after adjusting for pre-existing chronic disease status, the intervention group was still significantly more likely to be vaccinated in the intention-to-treat analysis (odds ratio [OR] 2.45; 95\% CI 1.28-4.68; $\mathrm{p}=0.007$ ) and the perprotocol analysis (OR 2.52; 95\% CI 1.32-4.82; $\mathrm{p}=0.005)$. There were no substantive differences in the vaccination uptake rates of participants between the two study years (see Supplementary Table).

The proportion of participants who initiated discussion about influenza vaccination with a healthcare professional was higher among participants in the intervention group 
$204(19.9 \% ; n=32)$ than in the standard care group $(13.1 \% ; n=21)$, but the difference was not

205 statistically significant $(\mathrm{p}=0.10)$. Of participants who did not receive influenza vaccination

206 during pregnancy ( $\mathrm{n}=271), 45$ reported that they had attempted to get vaccinated. A

207 significantly higher proportion of participants who attempted to get vaccinated were in the 208 intervention group $(82.2 \% ; n=37)$ than in the standard care group $(17.8 \% ; n=8)(p<0.001)$. If 209 participants who made the attempt had received the vaccination, the vaccination rate would 210 have been $44.1 \%(n=71)$ in the intervention group and $15 \%(n=24)$ in the standard care group 211 (RD 29.1\%, 95\% CI 19.6\%-38.6\%, p<0.001) (Table 3). At baseline, only 6.2\% (n=20) of 212 participants reported that a healthcare professional had discussed influenza vaccination with 213 them. At follow-up, 8.5\% $(\mathrm{n}=26)$ of participants reported that they were advised against 214 influenza vaccine by a healthcare professional, which included obstetricians $(n=11)$, general 215 practitioners $(\mathrm{n}=8)$, and nurses $(\mathrm{n}=7)$.

\section{$217 \quad 4.0$ Discussion}

218 The results of this study show that a brief, one-to-one education intervention for pregnant 219 women significantly increased maternal influenza vaccination. However, the vaccination rate 220 in the intervention group (21.1\%) was still substantially below the Healthy People 2020 target 221 vaccination rate of $80 \%$ [36]. This may be because other supportive vaccination practices (e.g., on-site vaccine availability and positive recommendations from their obstetric healthcare provider) were not in place. Pregnant women needed to obtain the vaccination from a private provider, which increased vaccination barriers. In obstetric settings where vaccination is readily available however, the effectiveness of brief education may be greater as the barriers that exist in our setting would be removed. Furthermore, when our participants did attempt to

227 get vaccinated, many were advised against vaccination by a healthcare professional or were unable to receive the vaccine. If these participants had received vaccination, the vaccination rate in the intervention group would have been approximately twice as high. 

vaccination is complex. Studies show that doctors and nurses frequently recommend influenza vaccination to elderly or chronically ill clients or people perceived to be at highest

233 risk from influenza morbidity and mortality [37-39]. However, healthcare professionals are

234 less likely to recommend vaccination for young healthy populations [40-42]. Furthermore, 235 rates of influenza vaccination among healthcare professionals, an identified risk group, are 236 consistently low [43-45]. Studies of US obstetric healthcare providers have found that over $23785 \%$ report that they routinely recommend influenza vaccine to their pregnant patients $[20,46$, 238 47]. Other studies however, suggest that many obstetric healthcare providers are unaware of 239 vaccine recommendations for pregnant women and even if aware, are reluctant to recommend 240 vaccination $[40,42,48,49]$. In addition, surveys of pregnant women have found that only $7-$ $24140 \%$ report receiving such a recommendation [32, 33, 50-52]. Although pregnant women who 242 receive a vaccination recommendation from their healthcare provider are substantially more 243 likely to receive influenza vaccination [14], only 30-70\% of pregnant women receiving the 244 recommendation get vaccinated [33, 50-52]. This suggests that even with knowledge of the 245 benefits of vaccination, many pregnant women remain reluctant to get vaccinated. This 246 reluctance is likely due to an long-held belief system that pregnant women should minimize 247 exposing the fetus to any unknown or potentially adverse substances [46], especially those 248 injected into the body. Evidence has shown that interventions targeting healthcare 249 professionals improved maternal influenza vaccination rates $[18,21,53]$. In our study a nurse 250 delivered the education intervention and recommended the vaccination to participants, and 251 although vaccine uptake was significantly improved, rates were still suboptimal. Pregnant 252 women may be more willing to follow recommendations from their regular GP or obstetric 253 healthcare provider but some women may still be reticent to receive the vaccination during 254 pregnancy [54]. In addition to maternal education, enthusiastic vaccination recommendations, 
and on-site vaccine access, vaccine promotion through mass media and social media may help

256

to further overcome these barriers [46].

In this study the vaccination coverage in the standard care group $(\sim 10 \%)$ was

somewhat higher than in previous Hong Kong studies among pregnant women, where rates

ranged from $1.7-6.2 \%[15,32,33]$. The influenza vaccination pamphlet provided to participants in the standard care group was widely available in antenatal clinics. However, it is not given directly to pregnant women, and it is likely that few read the pamphlet. Therefore, it is possible that simply being given the influenza vaccination pamphlet by a nurse increased the women's risk perceptions and perceived importance of vaccination. Other studies have shown significant increases in maternal influenza vaccination coverage following the distribution of education pamphlets by healthcare professionals [23, 55]. In addition, pregnant women may perceive healthcare staff-delivered information as more personally relevant and important [56]. Although the effect may be small, actively distributing pamphlets is a simple action, easily implemented in clinical settings at a minimal cost.

\subsection{Strengths and limitations}

This study provides high-quality evidence of the effectiveness of brief education in improving maternal influenza vaccination rates. First, random allocation and allocation concealment minimized treatment assignment bias. Second, there was a high participation rate. This might have been because the study involved only a brief onsite intervention, requiring less than 10 minutes of participants' time, and a short follow-up telephone interview. Evidence shows that people are more likely to participate in studies with a low participation burden such as inperson or telephone interviews [57]. Third, as the intervention was delivered immediately after randomization, we achieved $100 \%$ treatment fidelity. Finally, the loss to follow-up rate was $<5 \%$, meaning the risk of attrition bias was minimal. 
This study also has some limitations that need to be considered when interpreting the

281 findings. First, participants were recruited from the antenatal clinics at four public hospitals;

282 therefore, the demographic and socioeconomic characteristics might not be representative of

283 all pregnant women in Hong Kong. When compared with the 2014 Hong Kong female

284 population from 20-49 years of age, our sample had fewer participants in the lowest education

285 category (7.2\% vs. $17.9 \%)$ and more participants in the higher education category (42.4\% vs.

$28630.7 \%$ ) [58]. Second, the higher-than-expected vaccination rate in the standard care group

287 might indicate that study participants were more receptive to the influenza vaccination

288 information than other pregnant women. As the study information sheet, the consent form,

289 and the education pamphlet all identified that the study was on influenza vaccination, the

290 standard care group may have also received some priming regarding the importance of

291 influenza vaccine in pregnancy. Third, although we took measures to minimize potential

292 contamination between the two treatment groups, we did not assess whether there was

293 contamination or sharing of information between the participants. Fourth, the H7N9 avian

294 influenza outbreak may also explain the higher-than-expected vaccination rate in the standard

295 care group. However, outbreaks of avian influenza are not uncommon in Hong Kong [59] and

296 these outbreaks have had minimal impact on influenza vaccination rates in various population

297 and at risk groups [60, 61]. Fifth, it is also possible, as the assessment of the primary outcome

298 relied on self-reported data, reporting or recall bias may have affected the study results. It was

299 not possible to verify participants' vaccination status as most primary care providers work in

300 solo practices that do not have centralized vaccination reporting systems. However, existing

301 studies have shown that recall of vaccination status is accurate, and maternal recall is

302 particularly reliable for pregnancy-related events [62-64]. In addition, the unavailability of

303 influenza vaccine in the antenatal clinics may have limited the effect of antenatal education as

304 other barriers such as employment or lack of childcare may have prevented pregnant women

305 from being vaccinated. Finally, due to the nature of the intervention, participants and the 
research nurse could not be blinded to the treatment allocation and this may have biased the

study in some unmeasurable way.

\subsection{Conclusion}

310 Although our study supports the effectiveness of brief education in improving maternal

311 influenza vaccination rates, coverage remained low. It is possible that in populations with

312 higher baseline vaccination rates, brief education may be sufficient to achieve target

313 vaccination rates. However, in populations such as Hong Kong, where baseline vaccination

314 rates are low, multi-component interventions are likely required. In addition to education

315 about influenza vaccination, other supportive practices such as a direct healthcare professional

316 recommendation, onsite vaccination, and promotion campaigns that specifically address maternal concerns and fears about vaccination may need to be implemented to reduce barriers and achieve optimal vaccination coverage.

Full text of the trial protocol is available at www.biomedcentral.com/1471-2393/14/19 [28].

\section{Study Funding}

323 Funding for this study was provided by the Health and Medical Research Fund, Government

324 of the Hong Kong Special Administration Region, Hong Kong SAR (Grant No. 12111272).

Conflict of Interest Statement

327 The authors have no potential conflicts of interest to report.

\section{Acknowledgement}

330 The authors thank Ms. Vincci Chan for her assistance with study management and telephone 331 follow-up. 


\section{Figure Caption}

333 Figure 1: Flow diagram of participants through each stage of the study

334 
336 [1] Dodds L, McNeil SA, Fell DB, Allen VM, Coombs A, Scott J, et al. Impact of influenza exposure on rates of hospital admissions and physician visits because of respiratory illness among pregnant women. CMAJ. 2007;176:463-8.

[2] Jamieson DJ, Honein MA, Rasmussen SA, Williams JL, Swerdlow DL, Biggerstaff MS, et al. H1N1 2009 influenza virus infection during pregnancy in the USA. Lancet. 2009;374:451-8.

[3] Laibl VR, Sheffield JS. Influenza and pneumonia in pregnancy. Clin Perinatol. 2005;32:727-38.

[4] Callaghan WM, Chu SY, Jamieson DJ. Deaths from seasonal influenza among pregnant women in the United States, 1998-2005. Obstet Gynecol. 2010;115:919-23.

[5] Pasternak B, Svanstrom H, Molgaard-Nielsen D, Krause TG, Emborg HD, Melbye M, et al. Vaccination against pandemic A/H1N1 2009 influenza in pregnancy and risk of fetal death: cohort study in Denmark. BMJ. 2012;344:e2794.

[6] Baum U, Leino T, Gissler M, Kilpi T, Jokinen J. Perinatal survival and health after maternal influenza A(H1N1)pdm09 vaccination: A cohort study of pregnancies stratified by trimester of vaccination. Vaccine. 2015;33:4850-7.

[7] Fabiani M, Bella A, Rota MC, Clagnan E, Gallo T, D'Amato M, et al. A/H1N1 pandemic influenza vaccination: A retrospective evaluation of adverse maternal, fetal and neonatal outcomes in a cohort of pregnant women in Italy. Vaccine. 2015;33:2240-7.

[8] Steinhoff MC, Omer SB, Roy E, Arifeen SE, Raqib R, Dodd C, et al. Neonatal outcomes after influenza immunization during pregnancy: a randomized controlled trial. CMAJ. 2012;184:645-53.

[9] Zaman K, Roy E, Arifeen SE, Rahman M, Raqib R, Wilson E, et al. Effectiveness of maternal influenza immunization in mothers and infants. N Engl J Med. 2008;359:155564.

[10] World Health Organization. Vaccines against influenza WHO position paper November 2012. Wkly Epidemiol Rec. 2012;87:461-76.

[11] Bone A, Guthmann J-P, Nicolau J, Levy-Bruhl D. Population and risk group uptake of H1N1 influenza vaccine in mainland France 2009-2010: results of a national vaccination campaign. Vaccine. 2010;28:8157-61.

[12] Mereckiene J, Cotter S, Nicoll A, Levy-Bruhl D, Ferro A, Tridente G, et al. National seasonal influenza vaccination survey in Europe, 2008. Euro Surveill. 2008;13:23.

[13] Sammon CJ, McGrogan A, Snowball J, de Vries CS. Pandemic influenza vaccination during pregnancy: an investigation of vaccine uptake during the 2009/10 pandemic vaccination campaign in Great Britain. Hum Vaccin Immunother. 2013;9:917-23.

[14] Yuen CY, Tarrant M. Determinants of uptake of influenza vaccination among pregnant women - A systematic review. Vaccine. 2014;32:4602-13. 
[15] Tarrant M, Wu KM, Yuen CY, Cheung KL, Chan VH. Determinants of 2009 A/H1N1 influenza vaccination among pregnant women in Hong Kong. Matern Child Health J.

$375 \quad 2013 ; 17: 23-32$.

[16] Wilson RJ, Paterson P, Jarrett C, Larson HJ. Understanding factors influencing vaccination acceptance during pregnancy globally: A literature review. Vaccine. 2015;33:6420-9.

[17] Collins J, Alona I, Tooher R, Marshall H. Increased awareness and health care provider endorsement is required to encourage pregnant women to be vaccinated. Hum Vaccin Immunother. 2014;10:2922-9.

[18] Klatt TE, Hopp E. Effect of a best-practice alert on the rate of influenza vaccination of pregnant women. Obstet Gynecol. 2012;119:301-5.

[19] McCarthy EA, Pollock WE, Nolan T, Hay S, McDonald S. Improving influenza vaccination coverage in pregnancy in Melbourne 2010-2011. Aust N Z J Obstet Gynaecol. 2012;52:334-41.

[20] Panda B, Stiller R, Panda A. Influenza vaccination during pregnancy and factors for lacking compliance with current CDC guidelines. J Matern Fetal Neonatal Med. 2011;24:402-6.

[21] Sherman MJ, Raker CA, Phipps MG. Improving influenza vaccination rates in pregnant women. J Reprod Med. 2012;57:371-6.

[22] Wong VW, Lok KY, Tarrant M. Interventions to increase the uptake of seasonal influenza vaccination among pregnant women: A systematic review. Vaccine. 2016;34:20-32.

[23] Meharry P, Cusson R, Stiller R, Vázquez M. Maternal influenza vaccination: Evaluation of a patient-centered pamphlet designed to increase uptake in pregnancy. Matern Child Health J. 2014;18:1205-14.

[24] Stockwell MS, Westhoff C, Kharbanda EO, Vargas CY, Camargo S, Vawdrey DK, et al. Influenza vaccine text message reminders for urban, low-income pregnant women: a randomized controlled trial. Am J Public Health. 2014;104 Suppl 1:e7-e12.

[25] Moniz MH, Hasley S, Meyn LA, Beigi RH. Improving influenza vaccination rates in pregnancy through text messaging: a randomized controlled trial. Obstet Gynecol. 2013;121:734-40.

[26] Chamberlain AT, Seib K, Ault KA, Rosenberg ES, Frew PM, Cortes M, et al. Improving influenza and Tdap vaccination during pregnancy: A cluster-randomized trial of a multicomponent antenatal vaccine promotion package in late influenza season. Vaccine. 2015;33:3571-9.

[27] Frew PM, Saint-Victor DS, Owens LE, Omer SB. Socioecological and message framing factors influencing maternal influenza immunization among minority women. Vaccine. 2014;32:1736-44. 
[28] Wong VW, Fong DY, Tarrant M. Brief education to increase uptake of influenza vaccine among pregnant women: a study protocol for a randomized controlled trial.

413 BMC Pregnancy Childbirth. 2014;14:19.

414 [29] Panel on Health Services. Latest arrangement for non-local pregnant women giving 415 birth in Hong Kong. In: Food and Health Bureau, editor. Hong Kong 2012.

416 [30] Centre for Health Protection. Influenza vaccine safe and effective: A gift to your 417 baby. Hong Kong: Author; 2013.

418 [31] Centre for Health Protection. Scientific Committee on Vaccine Preventable Diseases: 419 recommendations on Seasonal Influenza Vaccination for the 2015/16 Season. In: Health 420 Do, editor. Hong Kong: Department of Health; 2015.

421 [32] Lau JT, Cai Y, Tsui HY, Choi KC. Prevalence of influenza vaccination and associated 422 factors among pregnant women in Hong Kong. Vaccine. 2010;28:5389-97.

423 [33] Yuen CYS, Fong DYT, Lee ILY, Sing C, Siu ES-m, Tarrant M. Prevalence and 424 predictors of maternal seasonal influenza vaccination in Hong Kong. Vaccine. 425 2013;31:5281-8.

426 [34] Faul F, Erdfelder E, Lang AG, Buchner A. G*Power 3: a flexible statistical power 427 analysis program for the social, behavioral, and biomedical sciences. Behav Res 428 Methods. 2007;39:175-91. [35] StataCorp. Stata Statistical Software: Release 14.1. College Station, Tx: StataCorp LP; 2015.

[36] Healthy People 2020. Immunization and infectious diseases data details. Office of Disease Prevention and Health Promotion; 2016.

[37] de Souto Barreto P, Lapeyre-Mestre M, Vellas B, Rolland Y. Indicators of influenza and pneumococcal vaccination in French nursing home residents in 2011. Vaccine. 2014;32:846-51.

436 [38] Ryu SY, Kim SH, Park HS, Park J. Influenza vaccination among adults 65 years or older: a 2009-2010 community health survey in the Honam region of Korea. Int J

438 Environ Res Public Health. 2011;8:4197-206.

439 [39] Kroneman MW, van Essen GA. Variations in influenza vaccination coverage among 440 the high-risk population in Sweden in 2003/4 and 2004/5: a population survey. BMC 441 Public Health. 2007;7:113.

442 [40] Broughton DE, Beigi RH, Switzer GE, Raker CA, Anderson BL. Obstetric health care 443 workers' attitudes and beliefs regarding influenza vaccination in pregnancy. Obstet 444 Gynecol. 2009;114:981-7.

445 [41] Arriola CS, Mercado-Crespo MC, Rivera B, Serrano-Rodriguez R, Macklin N, Rivera 446 A, et al. Reasons for low influenza vaccination coverage among adults in Puerto Rico, 447 influenza season 2013-2014. Vaccine. 2015;33:3829-35. 
[42] Mersereau P, Layton C, Smith L, Kendrick J, Mitchell E, Amoozegar J, et al. Prenatal 449 care providers and influenza prevention and treatment: Lessons from the field. Matern $450 \quad$ Child Health J. 2012;16:479-85.

451 [43] Lu PJ, Ding H, Black CL. H1N1 and seasonal influenza vaccination of U.S. healthcare personnel, 2010. Am J Prev Med. 2012;43:282-92.

453

454

455

456

457

458

459

460

461

462

463

464

465

466

467

468

469

470

471

472

473

474

475

476

477

478

479

480

481

482

483

484

[44] Zhang J, While AE, Norman IJ. Nurses' vaccination against pandemic H1N1 influenza and their knowledge and other factors. Vaccine. 2012;30:4813-9.

[45] Vaux S, Noel D, Fonteneau L, Guthmann JP, Levy-Bruhl D. Influenza vaccination coverage of healthcare workers and residents and their determinants in nursing homes for elderly people in France: a cross-sectional survey. BMC Public Health. 2010;10:159.

[46] Arao RF, Rosenberg KD, McWeeney S, Hedberg K. Influenza vaccination of pregnant women: attitudes and behaviors of Oregon physician prenatal care providers. Matern Child Health J. 2015;19:783-9.

[47] Power ML, Leddy MA, Anderson BL, Gall SA, Gonik B, Schulkin J. Obstetriciangynecologists' practices and perceived knowledge regarding immunization. Am J Prev Med. 2009;37:231-4.

[48] Maher L, Dawson A, Wiley K, Hope K, Torvaldsen S, Lawrence G, et al. Influenza vaccination during pregnancy: a qualitative study of the knowledge, attitudes, beliefs, and practices of general practitioners in Central and South-Western Sydney. BMC family practice. 2014;15:102.

[49] Tong A, Biringer A, Ofner-Agostini M, Upshur R, McGeer A. A cross-sectional study of maternity care providers' and women's knowledge, attitudes, and behaviours towards influenza vaccination during pregnancy. J Obstet Gynaecol Can. 2008;30:40410.

[50] Blanchard-Rohner G, Meier S, Ryser J, Schaller D, Combescure C, Yudin MH, et al. Acceptability of maternal immunization against influenza: the critical role of obstetricians. J Matern Fetal Neonatal Med. 2012;25:1800-9.

[51] Maher L, Hope K, Torvaldsen S, Lawrence G, Dawson A, Wiley K, et al. Influenza vaccination during pregnancy: coverage rates and influencing factors in two urban districts in Sydney. Vaccine. 2013;31:5557-64.

[52] Wiley KE, Massey PD, Cooper SC, Wood NJ, Ho J, Quinn HE, et al. Uptake of influenza vaccine by pregnant women: a cross-sectional survey. Med J Aust. 2013;198:373-5.

[53] Mouzoon ME, Munoz FM, Greisinger AJ, Brehm BJ, Wehmanen OA, Smith FA, et al. Improving influenza immunization in pregnant women and healthcare workers. Am J Manag Care. 2010;16:209-16.

[54] Yuen CY, Dodgson JE, Tarrant M. Perceptions of Hong Kong Chinese women toward influenza vaccination during pregnancy. Vaccine. 2016;34:33-40. 
[55] Yudin MH, Salaripour M, Sgro MD. Impact of patient education on knowledge of influenza and vaccine recommendations among pregnant women. J Obstet Gynaecol Can.

$487 \quad 2010 ; 32: 232-7$.

488 [56] Petty RE, Cacioppo JT. Attitudes and persuasion: classic and contemporary 489 approaches. Boulder, Colo.: Westview Press; 1996.

490 [57] Newington L, Metcalfe A. Factors influencing recruitment to research: qualitative 491 study of the experiences and perceptions of research teams. BMC Med Res Methodol. 492 2014;14:10.

493 [58] Census and Statistics Department. Women and men in Hong Kong: key statistics. 4942015 edition. Hong Kong: Hong Kong Census and Statistics Department; 2015.

495 [59] Parry J. Ten years of fighting bird flu. Bull World Health Organ. 2007;85:3-4.

496 [60] Yeung MP, Ng SK-C, Tong ETF, Chan SS-K, Coker R. Factors associated with uptake 497 of influenza vaccine in people aged 50 to 64 years in Hong Kong: a case-control study. 498 BMC Public Health. 2015;15:1-7.

499 [61] Mo PK, Lau JT. Influenza vaccination uptake and associated factors among elderly 500 population in Hong Kong: the application of the Health Belief Model. Health Educ Res. $501 \quad 2015 ; 30: 706-18$.

502 [62] Bat-Erdene U, Metcalfe A, McDonald SW, Tough SC. Validation of Canadian mothers' 503 recall of events in labour and delivery with electronic health records. BMC Pregnancy 504 Childbirth. 2013;13 Suppl 1:S3.

505 [63] Liu J, Tuvblad C, Li L, Raine A, Baker LA. Medical record validation of maternal recall 506 of pregnancy and birth events from a twin cohort. Twin Res Hum Genet. 2013;16:84550760 .

508 [64] Cupul-Uicab LA, Gladen BC, Hernandez-Avila M, Longnecker MP. Reliability of 509 reported breastfeeding duration among reproductive-aged women from Mexico. Matern 510 Child Nutr. 2009;5:125-37. 
Table 1. Baseline Characteristics of Participants by Intervention Group

\begin{tabular}{|c|c|c|c|c|}
\hline Demographic variable & $\begin{array}{c}\text { Standard care } \\
(\mathrm{n}=160), \\
\text { No. }(\%)\end{array}$ & 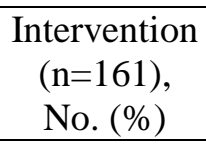 & Total $(n=321)$ & $P$ \\
\hline Maternal age, year $\mathrm{M}(\mathrm{SD})$ & $33.8 \pm 4.3$ & $33.2 \pm 4.0$ & $33.5 \pm 4.2$ & .21 \\
\hline \multicolumn{5}{|l|}{ Parity } \\
\hline 0 & $99(61.9)$ & $92(57.1)$ & $191(59.5)$ & \multirow[t]{3}{*}{.69} \\
\hline 1 & $53(33.1)$ & $60(37.3)$ & $113(35.2)$ & \\
\hline$>=2$ & $8(5.0)$ & $9(5.6)$ & $17(5.3)$ & \\
\hline \multicolumn{5}{|l|}{ Maternal education } \\
\hline Compulsory secondary or below & $12(7.5)$ & $11(6.8)$ & $23(7.2)$ & \multirow[t]{4}{*}{.21} \\
\hline Upper secondary & $64(40.0)$ & $68(42.2)$ & $53(41.1)$ & \\
\hline Some post-secondary & $10(6.3)$ & $20(12.4)$ & $30(9.4)$ & \\
\hline University degree or above & $74(46.3)$ & $62(38.5)$ & $136(42.4)$ & \\
\hline \multicolumn{5}{|l|}{ Place of birth } \\
\hline Hong Kong SAR & $116(72.5)$ & $112(69.6)$ & $228(71.0)$ & \multirow[t]{3}{*}{.71} \\
\hline Mainland China & $41(25.6)$ & $47(29.2)$ & $88(27.4)$ & \\
\hline Others & $3(1.9)$ & $2(1.2)$ & $5(1.6)$ & \\
\hline \multicolumn{5}{|l|}{ Length of residency in Hong Kong } \\
\hline$<10$ years & $20(12.5)$ & $21(13.0)$ & $41(12.8)$ & \multirow[t]{3}{*}{.75} \\
\hline $10-15$ years & $26(16.3)$ & $31(19.3)$ & $57(17.8)$ & \\
\hline Since birth & $114(71.3)$ & 109 (67.7) & $223(69.5)$ & \\
\hline \multicolumn{5}{|l|}{ Family income ${ }^{1}$} \\
\hline Below median & $44(27.5)$ & $49(30.4)$ & $93(29.0)$ & \multirow[t]{2}{*}{.56} \\
\hline Above median & $116(72.5)$ & $112(69.6)$ & $228(71.0)$ & \\
\hline \multicolumn{5}{|l|}{ Smoked during pregnancy } \\
\hline No & $158(98.8)$ & $157(97.5)$ & $315(98.1)$ & \multirow[t]{2}{*}{.69} \\
\hline Yes & $2(1.3)$ & $4(2.5)$ & $6(1.9)$ & \\
\hline \multicolumn{5}{|l|}{ Pre-existing chronic illness } \\
\hline No & $149(93.1)$ & $134(83.2)$ & $283(88.2)$ & \multirow[t]{2}{*}{.006} \\
\hline Yes & $11(6.9)$ & $27(16.8)$ & $38(11.8)$ & \\
\hline \multicolumn{4}{|l|}{$\begin{array}{l}\text { Types: (some participants had >1 } \\
\text { illness) }\end{array}$} & \multirow[t]{5}{*}{--} \\
\hline Hepatitis B carrier status & $3(27.3)$ & $11(40.7)$ & $14(36.8)$ & \\
\hline Respiratory disease & $1(9.1)$ & $5(18.5)$ & $6(15.8)$ & \\
\hline Thyroid disease & $1(9.1)$ & $5(18.5)$ & $6(15.8)$ & \\
\hline Others & $6(54.5)$ & $7(25.9)$ & $13(34.2)$ & \\
\hline \multicolumn{5}{|l|}{ Pregnancy related health problem } \\
\hline No & $125(78.1)$ & $126(78.3)$ & $251(78.2)$ & \multirow[t]{2}{*}{.98} \\
\hline Yes & 35 (21.9) & $35(21.7)$ & $70(21.8)$ & \\
\hline $\begin{array}{l}\text { Types: (some participants had }>1 \\
\text { health problem) }\end{array}$ & & & & -- \\
\hline Gestational diabetes & $13(37.1)$ & $19(54.3)$ & $32(45.7)$ & \\
\hline Anaemia & $15(42.9)$ & $13(37.1)$ & $28(40.0)$ & \\
\hline Hypertension & $2(5.7)$ & $4(11.4)$ & $6(8.6)$ & \\
\hline Others & $5(14.3)$ & $2(5.7)$ & $7(10.0)$ & \\
\hline
\end{tabular}

${ }^{\mathrm{T}}$ Median household income in HK in 2011 was $\$ 20,000$ to $\$ 24,999$ HKD per month (1USD=7.7HKD) 
Table 2. Observed Influenza Vaccine Uptake During Pregnancy by Treatment Group ${ }^{1}$

\begin{tabular}{lcccc}
\hline & \multicolumn{2}{c}{ Treatment group, $\mathrm{n}(\%)$} & & \\
\cline { 2 - 3 } & \multicolumn{1}{c}{ Standard care } & Intervention & $\mathrm{RD}, \%(95 \% \mathrm{CI})$ & $P$ \\
\hline Intention-to-treat analysis & & & & \\
Vaccinated & $16(10.0)$ & $34(21.1)$ & & \\
Non-vaccinated & $144(90.0)$ & $127(78.9)$ & $11.1(3.3-19.0)$ & 0.006 \\
& & & & \\
Per-protocol analysis & ${ }^{2}$ & & & \\
Vaccinated & $16(10.4)$ & $34(22.5)$ & & \\
Non-vaccinated & $138(89.6)$ & $117(77.5)$ & $12.1(3.9-20.3)$ & 0.004 \\
\hline
\end{tabular}

$\mathrm{RD}=$ Risk Difference; $\mathrm{CI}=$ Confidence Interval

${ }^{1}$ The actual influenza vaccine uptake rate among pregnant women

${ }^{2}$ In the standard care group, $n=160$. In the intervention group, $n=161$.

${ }^{3}$ In the standard care group, $n=154$. In the intervention group, $n=151$ 
Table 3. Expected Influenza Vaccine Uptake During Pregnancy by Treatment Groups ${ }^{1}$ $\frac{\text { Table 3. Expected Influenza Vaccine Uptake During Pregnancy by Treatment Groups }}{\text { Treatment group, } \mathrm{n}(\%)}$

Intention-to-treat analysis $^{2}$

Vaccinated Intervention

$\mathrm{RD}, \%(95 \% \mathrm{CI})$ $P$

Non-vaccinated $24(15.0)$

$71(44.1)$

$136(85.0) \quad 90(55.9)$

$29.1(19.6-38.6)$

$<0.001$

Per-protocol analysis ${ }^{3}$

Vaccinated

$71(47.0)$

Non-vaccinated $130(84.4)$ $80(53.0)$ $31.4(21.6-41.2)$ $<0.001$

$\mathrm{RD}=$ Risk Difference; $\mathrm{CI}=$ Confidence Interval

${ }^{1}$ The estimated influenza vaccination rate if participants who attempted to be vaccinated had received the vaccine

${ }^{2}$ In the standard care group, $\mathrm{n}=160$. In the intervention group, $\mathrm{n}=161$.

${ }^{3}$ In the standard care group, $n=154$. In the intervention group, $n=151$ 


\section{Figures}

Click here to download high resolution image

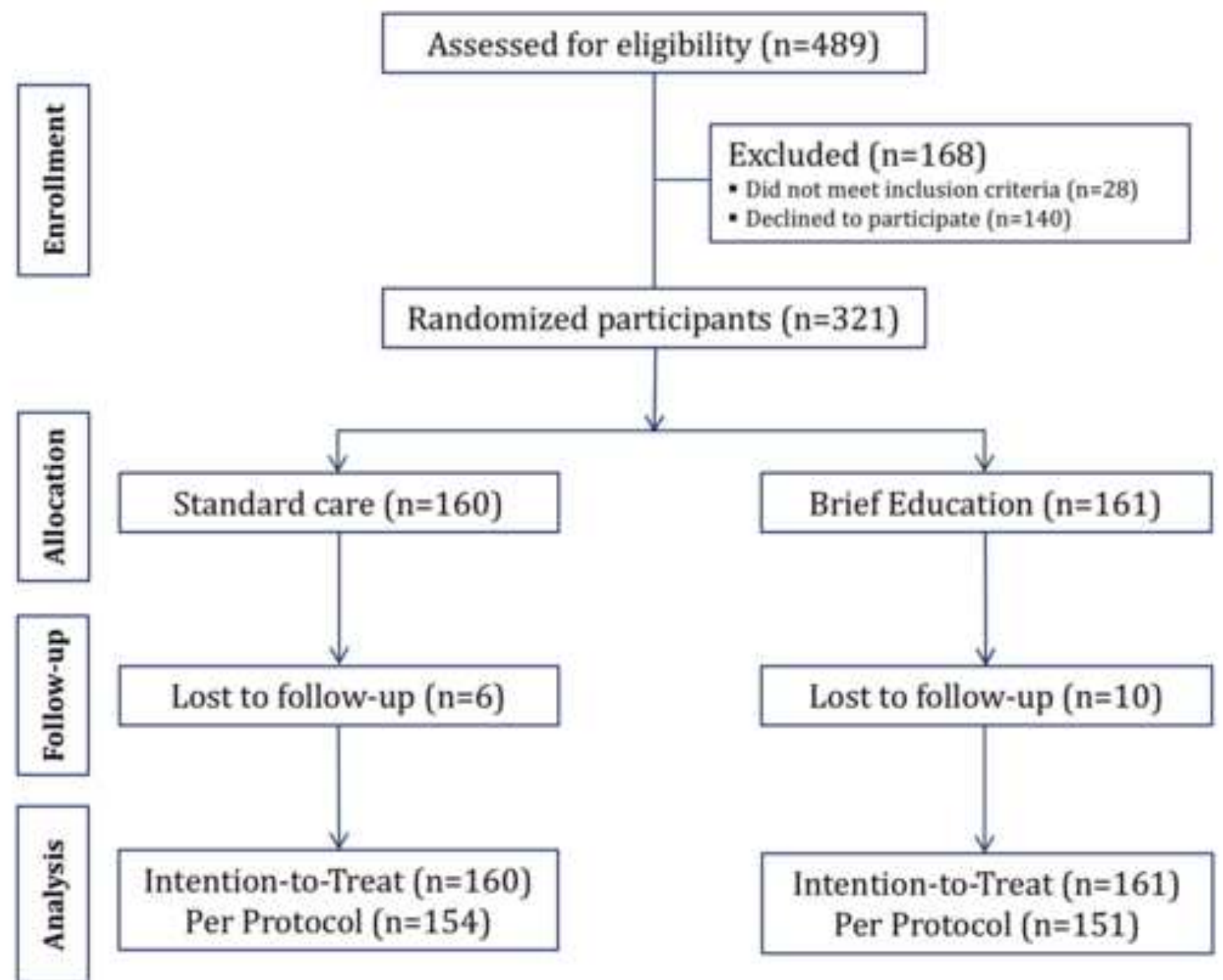

